\title{
Dendritic bundles, minicolumns, columns, and cortical output units
}

\author{
Giorgio M. Innocenti ${ }^{1}$ * and Alessandro Vercelli ${ }^{2}$ \\ 1 Department of Neuroscience, Karolinska Institutet, Stockholm, Sweden \\ 2 Neuroscience Institute, Medical School, University of Turin, Turin, Italy
}

Edited by:

Javier DeFelipe, Cajal Institute, Spain

Reviewed by:

Kathleen S. Rockland, Massachussetts Institute of Technology, USA

Alan Peters, Boston University School

of Medicine, USA

*Correspondence:

Giorgio M. Innocenti, Department of Neuroscience, Karolinska Institutet,

Retzius vag 8, S-17177 Stockholm,

Sweden.

e-mail: giorgio.innocenti@neuro.ki.se
The search for the fundamental building block of the cerebral cortex has highlighted three structures, perpendicular to the cortical surface: (i) columns of neurons with radially invariant response properties, e.g., receptive field position, sensory modality, stimulus orientation or direction, frequency tuning etc., (ii) minicolumns of radially aligned cell bodies and (iii) bundles, constituted by the apical dendrites of pyramidal neurons with cell bodies in different layers. The latter were described in detail, and sometimes quantitatively, in several species and areas. It was recently suggested that the dendritic bundles consist of apical dendrites belonging to neurons projecting their axons to specific targets. We review the concept above and suggest that another structural and computational unit of cerebral cortex is the cortical output unit, i.e., an assembly of bundles of apical dendrites and their parent cell bodies including each of the outputs to distant cortical or subcortical structures, of a given cortical locus (area or part of an area). This somato-dendritic assembly receives inputs some of which are common to the whole assembly and determine its radially invariant response properties, others are specific to one or more dendritic bundles, and determine the specific response signature of neurons in the different cortical layers and projecting to different targets.

Keywords: pyramidal neuron, cerebral cortex, apical dendrite, projection

\section{DENDRITIC BUNDLES: ORIGIN OF A CONCEPT}

Although the radial arrangement of dendrites (Fifkova, 1970) and cell bodies (von Bonin and Mehler, 1971) had been previously noticed (quoted in Peters and Walsh, 1972) the first comprehensive descriptions of dendritic bundles in cerebral cortex appear to be those of Peters and Walsh (1972) and of Fleischhauer et al. (1972). The work in Peters' laboratory was motivated by the search of a morphological substrate for the cortical "columns" initially demonstrated by physiological methods by Mountcastle (1957) in the primary somatosensory cortex, by Hubel and Wiesel (1963) in the primary visual cortex, and by Abeles and Goldstein Jr. (1970) in the primary auditory cortex. The study was carried out in tangential sections of the primary somatosensory cortex (area 3 ) of the rat, and later extended to the visual cortex (see below), and led to the following seminal concepts. (i) "Clusters" of apical dendrites are clearly visible in tangential sections through layers IV and III; (ii) the number of dendrites in a cluster varies between 1 and 14, the distance between clusters between 50 and $150 \mu \mathrm{m}$; (iii) the clusters correspond to dendritic bundles originating in layer $\mathrm{V}$, amplified by the addition of dendrites from more superficial neurons; (iv) the dendritic bundles correspond to aggregates of cell bodies visible in Nissl preparations. The work in Fleischhauer's laboratory was carried out in the sensory-motor cortex of rabbit and cat. The observations were compatible with what we summarized above, but with some emphasis on interarea and interspecies comparisons and differences (reviewed in Fleischhauer and Detzer, 1975).
The serial section reconstructions of Massing and Fleischhauer (1973) revealed some complications in the topographical organization of dendrites within a bundle. Individual dendrites changed their neighborhood relations along a bundle; superficial dendrites could be added between the dendrites from deeper layers; and individual dendrites could bifurcate to neighboring bundles. Subsequent work, reviewed in Peters (1997) and Rockland and Ichinohe (2004) refined some of the concepts above and extended them to a number of different species (next section). In addition to the bundles organized around the apical dendrites of layer V neurons, separate bundles of layer VI dendrites were described (Sakai, 1985; Escobar et al., 1986). Although dendritic bundles are most easily seen in layers III and IV, with appropriate methods they can also be identified in layer II (Miyashita et al., 2009). In the visual cortex the mean spacing between modules was found to be $60 \mu \mathrm{m}$ in the rat, $56 \mu \mathrm{m}$ in the cat and $23 \mu \mathrm{m}$ in the rhesus monkey (Peters, 1997). And the total number of bundles in the visual cortex was calculated to be 2.5 to $3.4 \times 10^{3}$ in the rat, $1.6 \times 10^{5}$ in the cat and $2.9 \times 10^{6}$ in the monkey. The physiological significance of the modules remained elusive, although their dimension would fit that of Mountcastle's (1997) minicolumns, and that of the orientation columns, at least in the monkey (see Section Functional Correlates of the Dendritic Bundles).

It was later found that myelinated axons are also organized in bundles; these bundles course close to those of the dendrites and at least some of them originate from neurons whose apical dendrites 
are in a bundle (Peters and Sethares, 1996). This is a first indication that neurons in a dendritic bundle might send their axons to the same target as it will be shown below.

\section{DENDRITIC BUNDLES ARE PRESENT BOTH ACROSS PHYLOGENESIS AND ONTOGENESIS}

In the cerebral cortex of mammals, dendritic bundles can be found across cortical areas, including area 17 (rat, Peters and Kara, 1987; cat, Peters and Yilmaz, 1993), somatosensory (rat, Peters and Walsh, 1972; mouse, White and Peters, 1993), motor (mouse, Lev and White, 1997; rabbit, Fleischhauer et al., 1972) and prelimbic (rat, Gabbott and Bacon, 1996) cortex, with quantitative features possibly reflecting differences in the density of neurons (rat, Skoglund et al., 1996) or locale. For example, in the barrelfield of rodent somatosensory cortex, dendritic bundles are mostly located in the barrel walls and septa, avoiding hollows (mouse, Escobar et al., 1986).

The appearance of vertically oriented dendritic bundles seems to be closely related to the evolution of the mammalian neocortex as a multilayered structure. Dendritic bundles have been found in the cerebral cortex of different mammalian species, such as rodents (mouse, Detzer, 1976; Escobar et al., 1986; rat, Peters and Walsh, 1972; Winkelmann et al., 1975; Peters and Kara, 1987; Gabbott and Bacon, 1996), lagomorphs (rabbit, Fleischhauer et al., 1972; Schmolke and Viebahn, 1986; Schmolke, 1996), carnivores (cat, Fleischhauer, 1974; Ikeda et al., 1989; Peters and Yilmaz, 1993) and primates (Peters and Sethares, 1991), including humans (von Bonin and Mehler, 1971). In the opossum they were reported as unpublished data by Peters and Feldman (1973). In the lesser hedgehog tenrec, a mammal bearing one of the lowest neocorticalization indices, dendritic bundles can be found in all cortical areas as well, including paleocortex (entorhinal cortex) and archicortex (hippocampus) (Schmolke and Künzle, 1997). On the contrary, vertically arranged dendritic bundles are not found in the primitive cortex of turtles (Schmolke and Künzle, 1997).

Dendritic bundles are described as early as E16, when the cortical plate forms in the rat parietal cortex (Hirst et al., 1991) and throughout development (Peters and Feldman, 1973; Schmolke, 1989; Hirst et al., 1991). According to Peters and Feldman (1973), in Nissl preparations, the cell bodies tend to align in vertical rows separated from each other by bundles (clusters) of vertically oriented processes, i.e., developing dendrites in the upper layers and axons, in lower layers. This is particularly evident at the top of the plate, where the lateral separation is wider than more deeply. The arrangement in vertical clusters is apparent at E19-E21. Vertical dendritic bundles (clusters) are masked in the mature cortex by the extensive proliferation of the neuropile.

The dendritic bundling, from neurons in layer II, was enhanced in the barrelfield by the over expression of the neurotrophin NT-2, an effect paralleled by an increased dendritic branching in layer I (Miyashita et al., 2009).

\section{METHODOLOGICAL AND DIMENSIONAL ISSUES}

The definition of a dendritic bundle raises a number of methodological issues. The histological methods used include material prepared for electron microscopy and viewed in thin or semithin sections, the Golgi technique, staining with microtubule associated protein 2 (MAP2), a selective somato-dendritic marker, retrograde transport of lipophilic tracers and intracellular injection in slice preparations. Depending on the sensitivity and resolution of the technique, bundles can vary in the number and size of the constituent dendrites, and be complicated by the addition of oblique and basal dendrites. Indeed, the diameter and number of dendrites in a bundle can vary in different reports from the same author. This stresses the objective difficulty of defining the limits of the bundle, which, depending also on tangential location and depth, can be more or less sharp. In several papers the distance between bundles is mentioned but not the statistical size of the sample, nor if corrections were introduced for the tissue shrinkage.

In our study (Vercelli et al., 2004), we intended to use the retrograde transport of lipophilic tracers, but because only a few dendrites are labeled from each site of tracer application we needed to define strict criteria to include apical dendrites in a bundle. To this end we preliminarily quantified aspects of dendritic bundles in the rat visual cortex, based on MAP2 immunostained material. To identify apical dendrites in tangential sections, we measured the size of apical dendrites in layer III in coronal sections, where they could be traced from the cell body and therefore could be distinguished from the oblique branches. Having obtained an average size of 1.1-1.44 $\mu \mathrm{m}$ (depending on age) for apical dendrites, we drew maps of apical dendrites in tangential, MAP2-immunoreacted sections through layer III.

We transformed these maps into sets of points, one for each dendrite, whose coordinates were used to (i) eliminate as outliers all dendrites which were further than the maximal nearest neighbor distance (NND); this was set at 5 or $6 \mu \mathrm{m}$ (depending on age) since $90 \%$ of measured NNDs between two dendrites in the same bundle fell in the range of $1-5$ or $1-6 \mu \mathrm{m}$ (depending on age); (ii) generate Dirichlet tessellation/Voronoi polygons for apical dendrites, to obtain objective, quantifiable criteria to consider them clustered (Figure 1). Briefly, a polygon was assigned to each point, corresponding to an apical dendrite, by joining the midpoints of the segments connecting the apical dendrite to its neighbors. High values $(>64 \%)$ in the coefficient of variation for Voronoi polygons are suggestive of a clustered distribution of points, and this was the case for apical dendrites; (iii) in the next step, we assigned a point to each dendritic bundle, to generate Voronoi polygons to analyze their tangential spatial distribution. This procedure allowed quantifying density, NNDs, $\mathrm{CV}$, average diameter, average center-tocenter distance, and number of dendrites/bundle. The coefficient of variation of Voronoi polygons for dendritic bundles was very low $(<36 \%)$, thus indicating a regular spatial distribution. The same procedure was used at all ages considered, with consistent results. By comparing data obtained at different ages, we observed that dendrites display a moderate increase in diameter between $\mathrm{P} 3$ and P30. Between those ages there is also a small increase in diameter of the bundles which seems more related to the increased dendritic size than to the number of dendrites in a bundle. There is a consistent increase in the NNDs between bundles, and a parallel decrease in the density of dendrites and dendritic bundles, probably reflecting the increase in the neuropil and glia.

This procedure produced smaller bundles than those observed by Peters and Kara (1987) in the same area and species, bearing 6-6.4 dendrites instead of 8 , at a higher density and with a smaller 


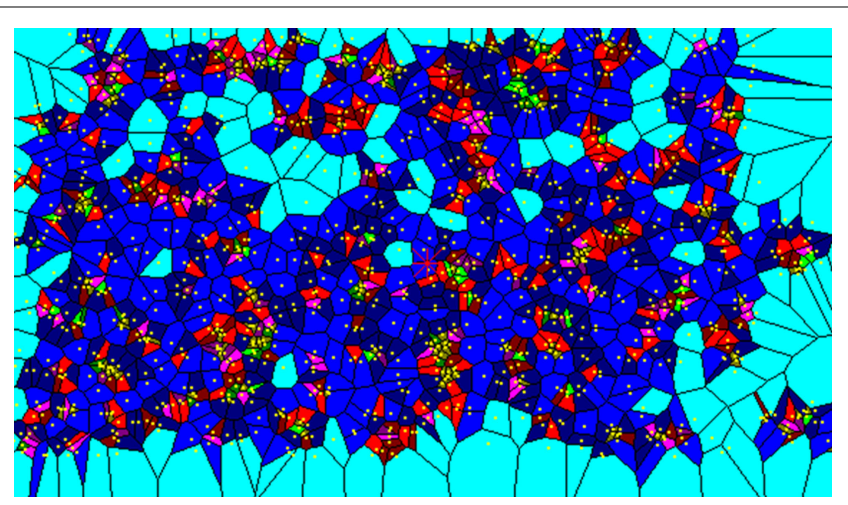

FIGURE 1 | Voronoi polygons drawn from tangential maps of MAP2positive apical dendrites. Each polygon area delineates the territory of the map which is closer to the point than to any other point of the map. Yellow dots correspond to apical dendrites. Colors of polygons are related to their size (green the smallest, light and dark blue the largest). The clusterization of dendrites is obvious.

NND. However, the two sets of results are only partially comparable, since (i) we considered dendrites of smaller size (1.1 instead of $2 \mu \mathrm{m}$ ), (ii) we drew our maps in layer III instead of layer IV, (iii) we considered bundles consisting of at least two apical dendrites instead of three, and (iv) we did not consider layer $\mathrm{V}$ apical dendrites to be essential in forming a bundle. Interestingly, our data are very similar to those obtained by other authors in area 17 and 18 of the monkey (Peters and Sethares, 1996; Peters et al., 1997), and in motor and somatosensory cortex of mice (Lev and White, 1997).

\section{SHIFTING CONCEPTS: DENDRITIC BUNDLES AS ASSEMBLIES OF TARGET-DEFINED NEURONS}

The meaning of the dendritic bundles underwent a potential fundamental shift with the work of Lev and White (1997). They showed that, in the mouse area MsI, following injection of horseradish peroxidase in the contralateral hemisphere, all dendrites in a labeled bundle belonged to callosally projecting neurons, thus suggesting that dendritic bundles are target-specific. The concept that different dendritic bundles may comprise neurons with different outputs is in keeping with the observed heterogeneity of dendritic bundles: bundles differ in the size of the constituent dendrites and that not all apical dendrites from layer $\mathrm{V}$ enter into the composition of dendritic bundles, as stressed by Rockland and Ichinohe (2004).

Because in the course of development some callosally projecting neurons lose their callosal axon and establish connections in the ipsilateral hemisphere (Innocenti et al., 1986; Clarke and Innocenti, 1990; reviewed in Innocenti and Price, 2005) it seemed likely that callosally and ipsilaterally projecting neurons would participate in the same dendritic bundle. Alternatively, the composition of the dendritic bundles might change in development.

Therefore we decided to identify, at different ages, pyramidal neurons in the visual cortex of the rat projecting to different targets by retrograde axonal tracers (Vercelli et al., 2004). We used DiI and DiA as tracers in the developing animal, and fluoro-emerald (FE) and fluoro-ruby (FR) in adults, labeling callosally projecting neurons with one dye and neurons projecting either to (i) the ipsilateral cortex, (ii) the superior colliculus, (iii) the pons, (iv) the lateral geniculate nucleus, (v) the striatum with the other dye. Moreover, in some animals we labeled corticopontine and corticostriatal neurons with the two dyes, respectively. Lipophilic dyes such as DiI and DiA are used to trace connections in vitro in fixed material thus allowing a precise placement of the dye even in deep subcortical targets and in small brains. On the other hand, these dyes hardly diffuse in adult material, so we were obliged to use in vivo tracing with FE and FR in adult rats. To maximize the chance of detecting double labeling in a bundle we analyzed regions with the highest density of neurons labeled with each tracer.

Compared with MAP2-immunostaining, retrograde tracing visualizes only a small number of neurons projecting to a target, proportional to the size of the crystal of lipophilic dyes or to the injection size for FE and FR. Also, retrograde tracing is not as efficient as MAP2 immunostaining in visualizing apical dendrites. Therefore, it was likely that not all the apical dendrites in the same bundle could be labeled retrogradely, implying that they could be at higher NND than in MAP2-immunostained sections. For this reason, we used the maximal diameter of MAP2-immunostained bundles $(28 \mu \mathrm{m})$ as cut-off distance to consider apical dendrites of neurons retrogradely labeled from different structures as participating in the same bundle.

Apical dendrites of neurons projecting to either corpus callosum, ipsilateral cortex or striatum as well as those projecting to pons and striatum were at a NND below this value indicating that they belonged to the same bundle. Of the 433 bundles, $30 \%$ containing apical dendrites of pyramidal neurons projecting to either ipsilateral or contralateral cortex contained both types of dendrites (Figures 2A,C, and 3), while 34\% contained only dendrites of neurons projecting to ipsilateral cortex and 30\% neurons projecting to contralateral cortex. In contrast, the distance between apical dendrites of neurons projecting to cortical targets, and of those projecting to subcortical targets, i.e., either superior colliculus, lateral geniculate or pons was never less than $30 \mu \mathrm{m}$, and peaked at 60 and $110 \mu \mathrm{m}$ suggesting that they belonged to different bundles. Of 551 bundles in brains in which callosally projecting and corticocollicular neurons were labeled, 81 and 19\% projected to either target, but none contained dendrites from both types of neurons (Figures 2B,D, and 3).

Our results are summarized in Figure 4. They strongly support the concept that dendritic bundles are target-specific. Moreover, the composition of dendritic bundles does not seem to depend on the age of the animal and is already established at P3.

\section{FUNCTIONAL CORRELATE OF THE DENDRITIC BUNDLES: THE CORTICAL OUTPUT UNIT HYPOTHESIS}

The search for the functional correlate of the dendritic bundles is of fundamental importance and has been the object of many speculations. One important preliminary question is that of the relation between the columns of radially aligned cell bodies, stained for example with the Nissl method, which have become known as minicolumns (Buxoeveden and Casanova, 2002), and the dendritic bundles. Dendritic bundles and minicolumns of cell bodies are closely related although not identical entities. The distance and the transverse diameter of both is roughly the same, i.e., about $50 \mu \mathrm{m}$ in most areas and species. In reconstructions from a limited series of tangential sections the dendritic bundles can be mapped onto 


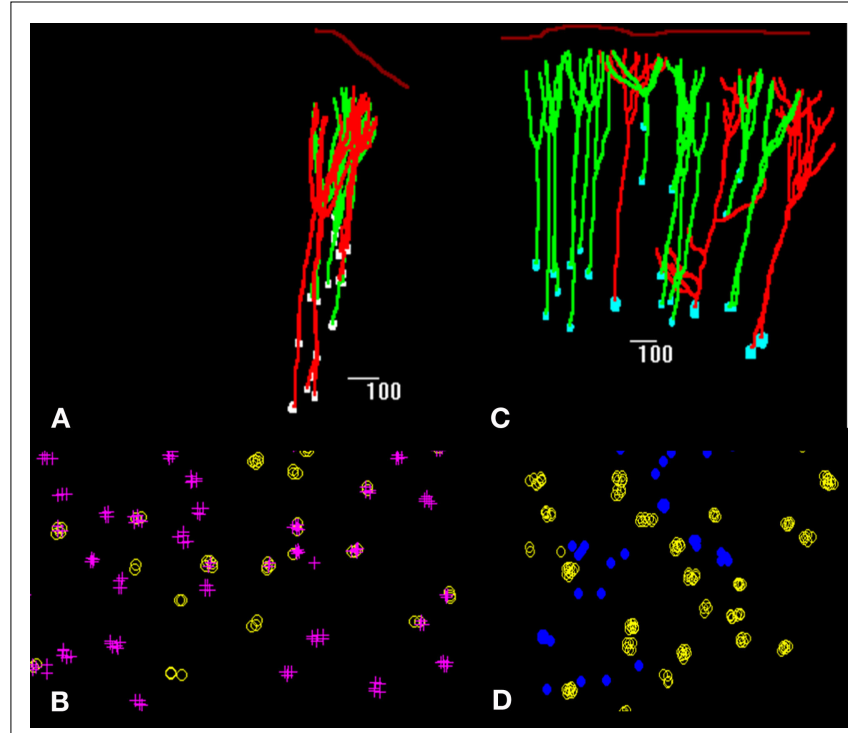

FIGURE 2 | Spatial distribution of retrogradely labeled pyramidal neurons in the rat visual cortex $(A, C)$ and tangential maps of apical dendrites $(B, D)$ drawn with Neurolucida software. In (A), neurons projecting to the corpus callosum in red and neurons projecting to the ipsilateral cortex in green; in (B) apical dendrites of callosally projecting neurons are identified by crosses, those of ipsilaterally projecting corticocortical neurons by yellow circles. In (C), neurons projecting to the corpus callosum in green and neurons projecting to the superior colliculus in red; in (D) apical dendrites of callosally projecting neurons are identified by yellow circles, those of corticocollicular neurons are identified by blue dots.

the underlying cell bodies of the minicolumn (Peters and Walsh, 1972; Peters and Kara, 1987). This shows that already in layer V and more so in layer III the dendritic bundles lie between the columns of cell bodies (see Figures 3, 7 and 11B in Peters and Kara, 1987). Indeed, as schematized in Figure 5A, cell bodies of neurons in a minicolumn can be seen to orient obliquely to engage their apical dendrite into the neighboring dendritic bundles already in layer V and more so in layer III (Peters and Walsh, 1972; Peters and Kara, 1987; Gabbott, 2003). Neurons in a minicolumn can send their apical dendrite to different bundles (Peters and Kara, 1987), some of them through bifurcating apical dendrites (Massing and Fleischhauer, 1973; Fleischhauer and Detzer, 1975), and neurons in separate minicolumns can send their dendrites to the same bundle (see Figure 3 in Peters and Kara, 1987). The progressive addition of dendrites to the bundle from depth to surface in cortex ("like onions held by their stem"; Peters and Kara, 1987) also indicates that the bundles collect dendrites from more than one minicolumn of cell bodies.

The evidence above is particularly relevant in view of our observation that the dendritic bundles consist of neurons with specific targets. At least nine types of target-specific bundles seem to exist (Figure 4) each of which, based on the MAP2 analysis contains 2-70 dendrites (6.5-8 on average, Peters and Kara, 1987; Vercelli et al., 2004). Since the minicolumns contain $80-100$ cell bodies, out of which probably 60-80 are pyramidal neurons (Buxoeveden and Casanova, 2002) it would be in theory possible that one minicolumn gives rise to a whole set of 9 , target specific bundles. However, the evidence quoted above

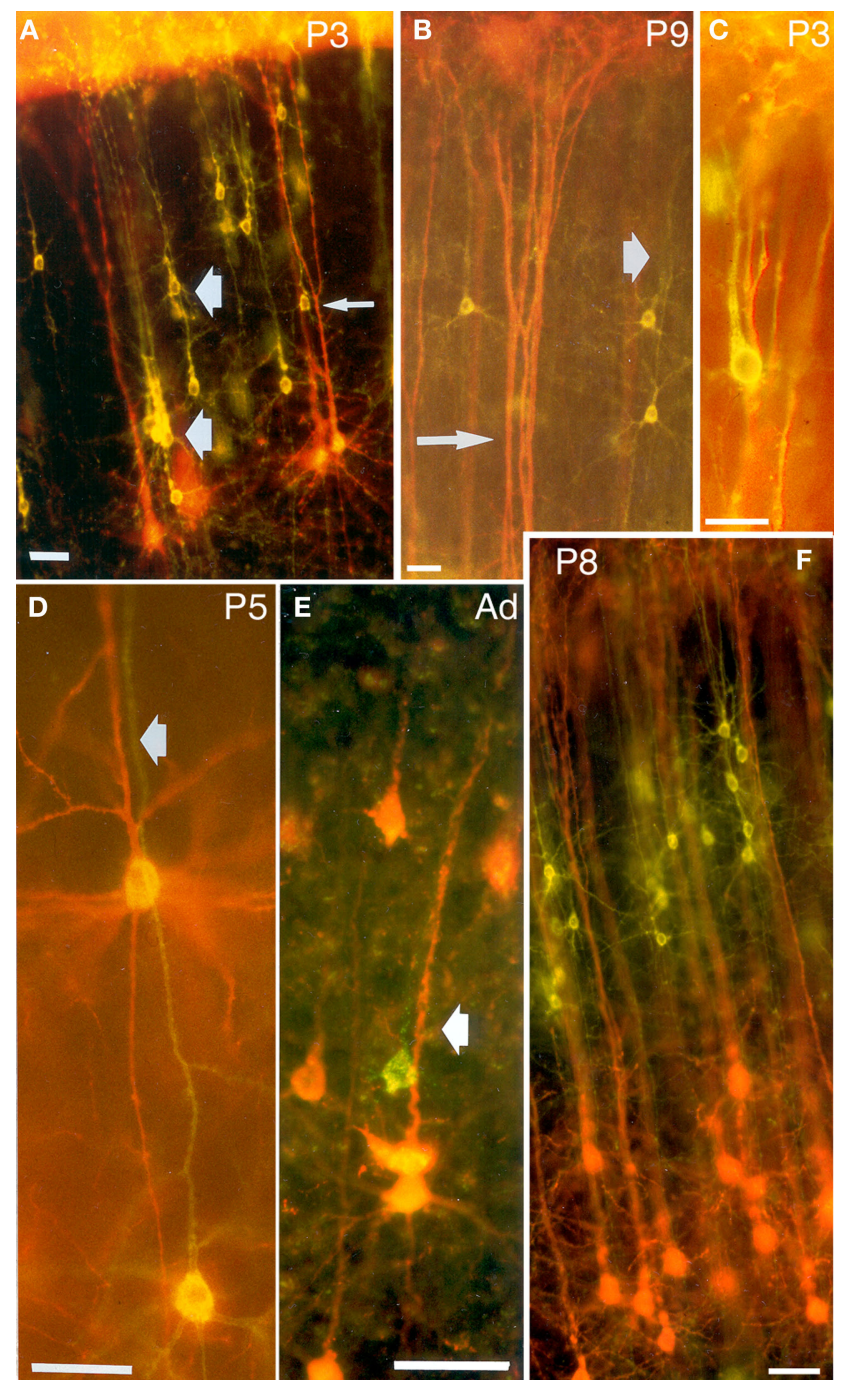

FIGURE 3 | Double exposures photomicrographs of coronal sections of double-labeled brains. In (A,B), callosally projecting (DiA, yellow-green) and corticocollicular (Dil, orange-red) neurons form separate bundles in the visual cortex of P3 and P9 rats. (A) Bundles are indicated by thick arrows, whereas the thin arrow points to a callosally projecting neuron whose apical dendrite, at higher magnification, was found to be separated from the corticocollicular ones on the z-axis. (B) The thin arrow points to a corticocollicular dendritic bundle, and the thick one to a callosally projecting bundle. (C) A bundle comprising apical dendrites of callosally projecting (Dil, orange-red) and corticostriatal neurons (DiA, yellow-green), in a P3 rat. (D,E) callosally projecting (Dil and fluoro-ruby, respectively, orange-red) and ipsilaterally projecting corticocortical (DiA and fluoro-emerald, respectively, yellow-green) neurons in the visual cortex of P5 and adult rats establish common bundles (thick arrows). (F) Callosally projecting (DiA, yellow-green) and corticogeniculate (Dil, orange-red) neurons in the visual cortex of P8 rats. Scale bars, $50 \mu \mathrm{m}$ (from Vercelli et al., 2004).

suggests that this is not the case, and that, instead, the bundles are formed both by dendrites diverging from neurons to more than one bundle as well as by convergence of dendrites whose parent cell bodies lie in separate minicolumns. The dendritic bundles, therefore, appear to select neuronal subsets within one minicolumn of cell bodies and integrate them with those of neighboring minicolumns. 


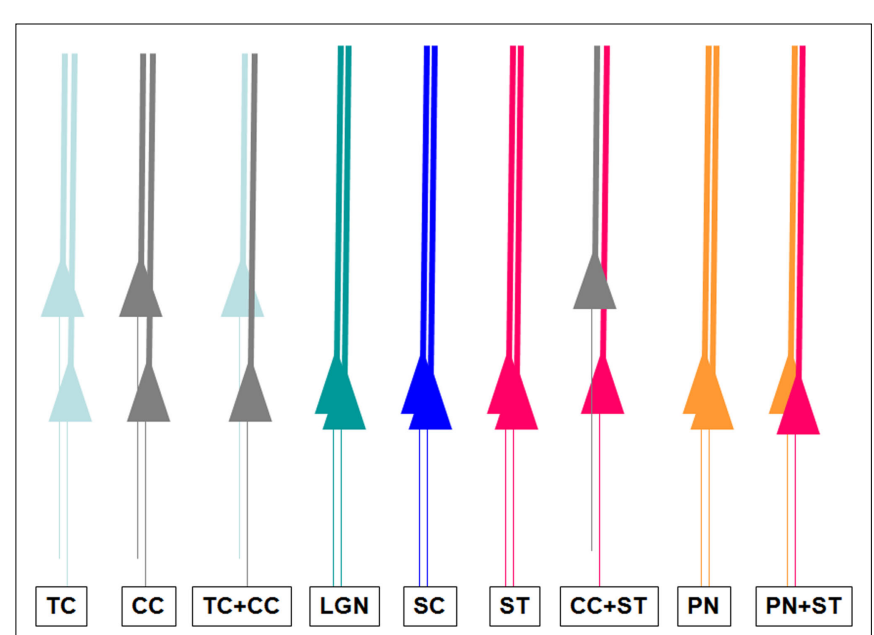

FIGURE 4 | Summary of the different types of dendritic bundles in the visual cortex, related to the axonal targets of their cell body. TC: neurons projecting to the ipsilateral cortex; CC: callosally projecting neurons; LGN: corticothalamic neurons; SC: corticocollicular neurons; ST: corticostriatal neurons; PN: corticopontine neurons.

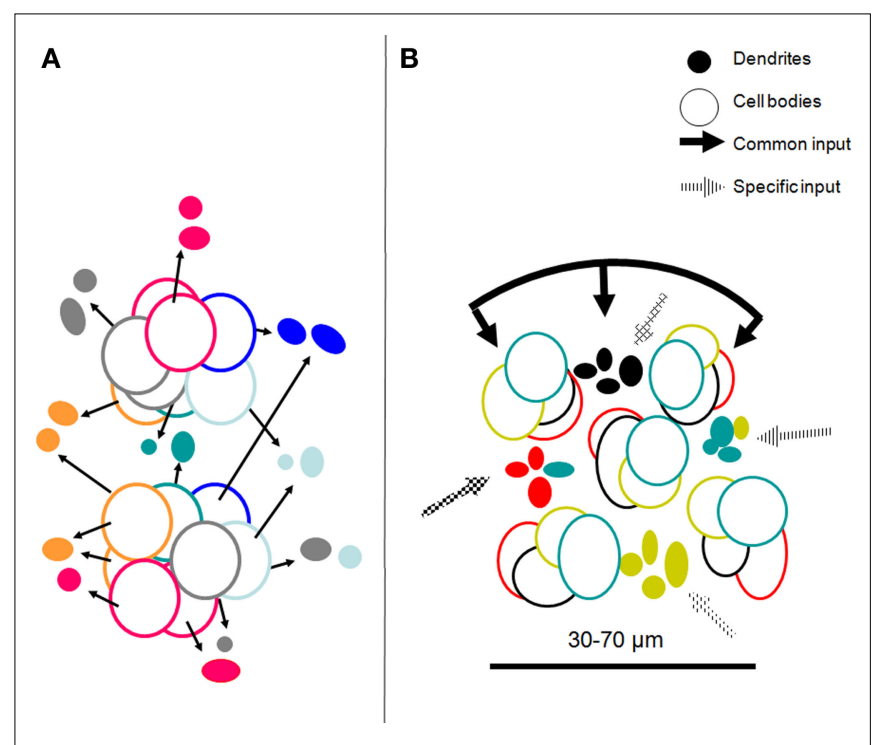

FIGURE 5 | (A) Topographical relations between minicolumns and dendritic bundles summarizing information from Massing and Fleischhauer (1973), Peters and Kara (1987; inspired by their Figure 3) and Vercelli et al. (2004). Dendrites are shown as "clusters" as they appear in transverse sections; the cell body outlines belong to neurons in minicolumns adjacent to the bundles; they are in different layers but are projected on the plane of the dendrites. The colors refer to specific targets or combinations of targets for each dendritic bundle and their parent somata (see Figure 4). (B) Model of a $\mathrm{COU}$ in layer III of $\mathrm{V} 1$ with bundles of apical dendrites of neurons projecting to four different targets (colors). The COU is defined as an assembly of apical dendritic bundles, large enough to include each of the outputs to distant cortical or subcortical structures, of a given cortical locale (area or part of an area), their parent somata and basal dendrites, and the portion of the neuropil which pertains to them. It receives a common input to the apical and basal (not shown) dendrites as well as specific inputs to the different dendritic bundles. The distance between the dendritic bundles and between minicolumns $30-70 \mu \mathrm{m}$ is similar and corresponds to the minimal transverse diameter of an orientation column in the visual cortex, estimated electrophysiologically.
The search for the functional correlate of the dendritic bundles is constrained by the following evidence.

From the time of their discovery, the dimension of the dendritic bundles, about $50 \mu \mathrm{m}$ (or less in the visual cortex of the monkey; see above) excluded that they might correspond to the large cortical columns, i.e., Hubel and Wiesel's ocular dominance columns or Mountcastle's receptive-field and tactile modality columns which average about $500 \mu \mathrm{m}$ in diameter (reviewed in Mountcastle, 1997). The possibility was raised that they might correspond to narrower assemblies of neurons with radially invariant activation/response properties such as orientation (and direction) of stimulus motion, in the visual, motor and auditory areas.

A microelectrode driven into the cortex perpendicularly to the cortical surface will, as a rule, record activity from neurons at 50-100 $\mu \mathrm{m}$ from its tip, therefore collecting responses of neurons which belong to the same minicolumn of cell bodies as well as to different minicolumns. Even when a microelectrode records simultaneously from nearby neurons, these very often share some response properties but differ for others (Creutzfeldt et al., 1974; Molotchnikoff et al., 2007; Sato et al., 2007; Dahl et al., 2009), suggesting that they receive a common input, but each of them also distinct ones. Nearby neurons have largely overlapping basal dendritic arbors which could receive common input but their apical dendrites can segregate to different bundles (Krieger et al., 2007) which might receive distinct inputs.

Although some response properties of cortical neurons are radially invariant, and therefore must be determined by an input which, directly or indirectly, reaches neurons in different layers, many are not. In particular, receptive field structure and size in the visual cortex vary with cortical depth (Hubel and Wiesel, 1962; Gilbert, 1977) and are different in neurons projecting to different targets (Swadlow and Weyand, 1987; Niida et al., 1997). This could be easily achieved by a differential distribution of inputs to dendritic bundles containing different sets of output neurons.

Finally, the evidence that the radially invariant properties of cortical neurons are the result of intracolumnar computation is lacking. In fact at least one of the response properties used to define a cortical column, i.e., orientation specificity is preserved in spite of the deletion of the deep cortical layers V and VI (Innocenti et al., 1993). Orientation specificity most probably originates from spatially organized excitatory input reaching several output neurons, possibly sharpened by inhibition (Hubel and Wiesel, 1962; Wörgötter and Koch, 1991; Crook et al., 1998; Ferster and Miller, 2000).

We propose that neurons in the different layers of one minicolumn, projecting to different targets, send their apical dendrites to separate dendritic bundles where they join apical dendrites of neurons from neighboring minicolumns, projecting to the same target or combination of targets. An assembly of apical dendritic bundles, which includes each of the outputs to distant cortical or subcortical structures, of a given cortical locale (area or part of an area), their parent somata and basal dendrites, and the portion of the neuropil which pertains to them, constitutes a cortical output unit (COU). We assume that the COU receives excitatory and inhibitory afferents some of which common to all its constituent neurons in particular those reaching the dendritic tufts in layer I or the largely overlapping basal dendrites (Krieger et al., 2007). Other inputs are probably specific for a given bundle and therefore reach 
specific sets of output neurons in the same or in different layers, determining their specific response properties (above). The dendritic bundling seems to offer two important advantages. It might minimize the length of the axonal arbors which contact specific neuronal classes and, in development it might simplify the axonal search and recognition of targets.

This view is summarized in the model shown in Figure 5B. The properties of the COU, match several aspects of Mountcastle (1997) definition of a cortical column: "A cortical column is a complex processing and distributing unit that links a number of inputs to a number of outputs via overlapping internal processing chains. Cortical efferent neurons with different extrinsic targets are partially segregated; those of layers II/III project to other cortical areas, those of layers V/VI to subcortical structures. This suggests that the intracolumnar processing operations leading to those different output channels may differ in some fundamental way".

Unfortunately, the internal connectivity of the COU is incompletely known. Unlike what might have been expected, neurons of the same bundle are not more interconnected than neurons of different bundles (Krieger et al., 2007). Instead, there are preferential connections between clonally related neurons, presumably belonging to the same minicolumn (Yu et al., 2009). There are also preferential connections between certain output neurons, interestingly between corticocortical and cortico-tectal neurons, whose apical dendrites, as we have described, lie in separate dendritic bundles (mice, Brown and Hestrin, 2009).

\section{MINICOLUMNS OF CELL BODIES, DENDRITIC BUNDLES, CLONES AND GENES}

One strong appeal of the hypothesis that minicolumns might be the fundamental computational unit in the cerebral cortex is that it appears to link cortical morphology and function to development, since the minicolumns of cell bodies seemed to be the likely counterpart of Rakic's ontogenetic radial units (Rakic, 1988; discussed in Buxoeveden and Casanova, 2002). Thus, there might be a direct path from the genetic make-up of clonally related neurons,

\section{REFERENCES}

Abeles, M., and Goldstein, M.H.Jr. (1970). Functional architecture in cat primary auditory cortex: columnar organization and organization according to depth. J. Neurophysiol. 33, 172-187.

Brown, S. P., and Hestrin, S. (2009). Intracortical circuits of pyramidal neurons reflect their long-range axonal targets. Nature 457, 1133-1137.

Buxoeveden, D. P., and Casanova, M. F. (2002). The minicolumn hypothesis in neuroscience. Brain 125, 935-951.

Clarke, S., and Innocenti, G. M. (1990). Auditory neurons with transitory axons to visual areas form short permanent projections. Eur. J. Neurosci. 2, 227-242.

Creutzfeldt, O., Innocenti, G. M., and Brooks, D. (1974). Vertical organization in the visual cortex (Area 17) in the cat. Exp. Brain Res. 21, 315-336.

at least the pyramidal neurons, to their connectivity and response properties although these are further refined by activity. The evidence for lateral dispersion leading to clonal intermingling among radially aligned neurons (Torii et al., 2009) complicates this perspective. However, a more direct link between cortical morphology, function and development, applies to dendritic bundles. Newly generated neurons reach their final position in cortex by ascending with their apical dendrites in close contact to the radial glia (Rakic, 1988). It would not be surprising if neurons which select the same radial glia processes because of some genetically determined membrane signaling properties, would also, in adulthood, participate in the same dendritic bundle whereby receiving the same input, while with their axons they participate in the same axonal bundle, proceeding to specific pathways and targets.

\section{PERSPECTIVES}

The hypothesis that assemblies of dendritic bundles, and minicolumns of cell bodies constitute COUs, and that these in turn might represent computational building blocks of neocortex requires several structural and physiological refinements. The retrograde tracing experiments mentioned in this paper could not adequately describe the frequency and spatial arrangements of the different target-specific dendritic bundles and the related minicolumns. Therefore, the model shown in Figure 5B is tentative and is meant to illustrate a concept the details of which are still fuzzy. Moreover, the arrangements might be area-specific, and might differ e.g. between the primary areas and/or between primary and secondary-association areas. The type and origin of the inputs to the different components of the COU needs specifying. The relations between COUs and the classical cortical macro-columns needs clarifying as does the clonal origin and the genetic make up of the neurons participating in the bundles.

\section{ACKNOWLEDGMENT}

Supported by Compagnia di San Paolo grant to Alessandro Vercelli.

of apical dendrites in the murine neocortex: dual apical dendritic systems. Neuroscience 17, 975-989.

Ferster, D., and Miller, K.D. (2000). Neural mechanisms of orientation selectivity in the visual cortex. Annu. Rev. Neurosci. 23, 441-471.

Fifkova, E. (1970). The effect of unilateral deprivation on visual centers in rats. J. Comp. Neurol. 140, 431-438.

Fleischhauer, K. (1974). On different patterns of dendritic bundling in the cerebral cortex of the cat. Z. Anat. Entwickl. Gesch. 143, 115-126.

Fleischhauer, K., and Detzer, K. (1975). Dendritic bundling in the cerebral cortex. In Advances in Neurology, G. W. Kreutzberg, ed. (New York, Raven Press).

Fleischhauer, K., Petsche, H., and Wittkowski, W. (1972). Vertical bundles of dendrites in the neocortex.
Z. Anat. Entwicklungsgesch. 136, 213-223.

Gabbott, P.L. (2003). Radial organisation of neurons and dendrites in human cortical areas 25, 32, and 32'. Brain Res. 992, 298-304.

Gabbott, P. L. A., and Bacon, S. J. (1996). The organization of dendritic bundles in the prelimbic cortex (area 32) of the rat. Brain Res. 730, 75-86.

Gilbert, C. D. (1977). Laminar differences in receptive field properties of cells in cat primary visual cortex. J. Physiol. 268, 391-421.

Hirst, E., Asante, J., and Price, J. (1991). Clustering of dendrites in the cerebral cortex begins in the embryonic cortical plate. J. Neurocytol. 20, 431-438.

Hubel, D. H., and Wiesel, T. N. (1962). Receptive fields, binocular interaction and functional architecture in 
the cat's visual cortex. J. Physiol. 160, 106-154.

Hubel, D. H., and Wiesel, T. N. (1963). Shape and arrangement of columns in cat's striate cortex. J. Physiol. 165, 559-568.

Ikeda, J., Mori, K., Oka, S., and Watanbe, Y. (1989). A columnar arrangement of dendritic processes of entorhinal cortex neurons revealed by a monoclonal antibody. Brain Res. 505, 176-179.

Innocenti, G. M., Berbel, P., and Assal, F. (1993). Anatomical and functional aspects of an experimental visual microcortex that resembles human microgyria. In Dyslexia and Development, A. M. Galaburda, ed. (Cambridge, MA, Harvard University Press), pp. 112-132.

Innocenti, G. M., Clarke, S., and Kraftsik, R. (1986). Interchange of callosal and association projections in the developing visual cortex. J. Neurosci. 6, 1384-1409.

Innocenti, G. M., and Price, D. J. (2005). Exuberance in the development of cortical networks. Nat. Rev. Neurosci. 6, 955-965.

Krieger, P., Kuner, T., and Sakmann, B. (2007). Synaptic connections between layer $5 \mathrm{~B}$ pyramidal neurons in mouse somatosensory cortex are independent of apical dendrite bundling. J. Neurosci. 27, 11473-11482.

Lev, D. L., and White, E. L. (1997). Organization of pyramidal cell apical dendrites and composition of dendritic clusters in the mouse: emphasis on primary motor cortex. Eur. J. Neurosci. 9, 280-290.

Massing, W., and Fleischhauer, K. (1973). Further observations on vertical bundles of dendrites in the cerebral cortex of the rabbit. $Z$. Anat. Entwicklungsgesch. 141, 115-123.

Miyashita, T., Wintzer, M., Kurotani, T., Konishi, T., Ichinohe, N., and Rockland, K. S. (2009). Neurotrophin3 is involved in the formation of apical dendritic bundles in cortical layer 2 of the rat. Cereb. Cortex 20, 229-240.
Molotchnikoff, S., Gillet, P.-C., Shumikhina,S., and Bouchard, M. (2007). Spatial frequency characteristics of nearby neurons in cats' visual cortex. Neurosci. Lett. 418, 242-247.

Mountcastle, V. B. (1957). Modality and topographic properties of single neurons of cat's somatic sensory cortex. J. Neurophysiol. 20, 408-434.

Mountcastle, V. B. (1997). The columnar organization of the neocortex. Brain $120,701-722$.

Niida, T., Stein, B., and McHaffie, J. G. (1997). Response properties of corticotectal and corticostriatal neurons in the postero lateral suprasylvian cortex of the cat. J. Neurosci. 17, 8550-8565.

Peters, A. (1997). Pyramidal cell modules in cerebral cortex. In: Recent Advances in Microscopy of Cells, Tissues and Organs, P. M. Motta, ed. (Rome, A. Delfino), pp. 187-192.

Peters, A., Cifuentes, J. M., and Sethares, C. (1997). The organization of pyramidal cells in area 18 of the rhesus monkey. Cereb. Cortex 7, 405-421.

Peters, A., and Feldman, M. (1973). The cortical plate and molecular layer of the late rat fetus. Z. Anat. Entwicklungsgesch. 141, 3-37.

Peters, A., and Kara, D. A. (1987). The neuronal composition of area 17 of rat visual cortex. IV. The organization of pyramidal cells. J. Comp. Neurol. 260, 573-590.

Peters, A., and Sethares, C. (1991). Organization of pyramidal neurons in area 17 of monkey visual cortex. J. Comp. Neurol. 306, 1-23.

Peters, A., and Sethares, C. (1996). Myelinated axons and the pyramidal cell modules in monkey primary visual cortex. J. Comp. Neurol. 365, 232-255.

Peters, A., and Walsh, M.A. (1972). Study of the organization of apical dendrites in the somatic sensory cortex of the rat. J. Comp. Neurol. 144, 253-268.

Peters, A., and Yilmaz,E. (1993). Neuronal organization in area 17 of cat visual cortex. Cereb. Cortex 3, 49-68.
Rakic, P. (1988). Specification of cerebral cortical areas. Science 241, 170-176.

Rockland, K. S., and Ichinohe, N. (2004). Some thoughts on cortical minicolumns. Exp. Brain Res. 158, 265-277.

Sakai, M. (1985). Dendritic bundles formed by layer VI pyramidal cells in the monkey frontal association cortex. Exp. Brain Res. 58, 609-612.

Sato, T.R., Gray, N.W.,Zachary,F., Mainen, Z. F., and Svoboda, K. (2007). The functional microarchitecture of the mouse barrel cortex. PLoS Biol. 5, e189. doi: 10.1371/journal.pbio.0050189.

Schmolke, C. (1989). The ontogeny of dendrite bundles in rabbit visual cortex. Anat. Embryol. 180, 371-381.

Schmolke, C. (1996). Tissue compartments in laminae II/V of rabbit visual cortex three dimensional arrangement, size and developmental changes. Anat. Embryol. 193, 15-33.

Schmolke, C., and Künzle, H. (1997). On the presence of dendrite bundles in the cerebral cortex of the Nadagascan lesser hedgehog tenrec and the redeared pond turtle. Anat. Embryol. 196, 195-213.

Schmolke, C., Viebahn, C. (1986). Dendrite bundles in lamina II/III of the rabbit neocortex. Anat. Embryol. 173, 343-348.

Skoglund, T. S., Pascher, R., and Berthold, C.-H. (1996). Heterogeneity in the columnar number of neurons in different neocortical areas in the rat. Neurosci. Lett. 208, 97-100.

Swadlow, H.A., and Weyand, T.G. (1987) Corticogeniculate neurons, corticotectal neurons and suspected interneurons in visual cortex of awake rabbits: receptive field properties, axonal properties and effects on EEG arousal. $J$. Neurophysiol. 57, 977-1001.

Torii, M., Hashimoto-Torii, K., Levitt, P., and Rakic, P. (2009). Integration of neural clones in the radial cortical columns by EphA and ephrin-A signalling. Nature 461, 524-528.

Vercelli, A., Garbossa, D., Curtetti, R., and Innocenti, G. M. (2004)
Somatodendritic minicolumns of output neurons in the rat visual cortex. Eur. J. Neurosci. 20, 495-502.

von Bonin, G., and Mehler, W. R. (1971) On columnar arrangement of nerve cells in cerebral cortex. Brain Res. 27, $1-9$.

White, E. L., and Peters, A. (1993). Cortical modules in the posteromedial barrel subfield (Sm1) of the mouse. J. Comp. Neurol. 334, 86-96.

Winkelmann, E., Brauer, K., and Berger, U. (1975).Zur columnaren organisation von Pyramidenzellen im visuellen cortex der Albinoratte. Z. Mikrosk. Anat. Forsch. 2, 239-256.

Wörgötter, F., and Koch, C. (1991). A detailed model of the primary visual pathway in the cat. Comparison of afferent excitatory and intracortical inhibitory connection schemes for orientation selectivity. I. Neurosci. 11, 1959-1979.

Yu, Y.-C., Bultje, R. S., Wang, X., and Shi, S.-H. (2009). Specific synapses develop preferentially among syster excitatory neurons in the neocortex. Nature 458, 501-505.

Conflict of Interest Statement: The authors declare that the research was conducted in the absence of any commercial or financial relationships that could be construed as a potential conflict of interest.

Received: 06 December 2009; paper pending published: 15 January 2010; accepted: 06 February 2010; published online: 12 March 2010.

Citation:InnocentiGMand VercelliA (2010) Dendritic bundles, minicolumns, columns, and cortical output units. Front. Neuroanat. 4:11. doi: 10.3389/neuro.05.011.2010 Copyright (c) 2010 Innocenti and Vercelli. This is an open-access article subject to an exclusive license agreement between the authors and the Frontiers Research Foundation, which permits unrestricted use, distribution, and reproduction in any medium, provided the original authors and source are credited. 